\title{
First Do Less Harm: Confronting the Inconvenient Problems of Patient Safety
}

Edited by

Ross Koppel and Suzanne Gordon

Reviewed by

Richard Wray RN, BA, MN

Director, Quality, Safety, Infection Prevention \& Control The Hospital for Sick Children, Toronto, ON

CONFRONTING

THE INCONVENIENT

PROBLEMS OF

PATIENT SAFETY 
First Do Less Harm: Confronting the Inconvenient Problems of Patient Safety is a diverse collection of perspectives that tell the story of a complex healthcare system, revealing correspondingly complex issues that have slowed the advancement of patient safety to a seemingly glacial pace. The story unfolds through a series of freestanding and intertwined points of view presented in chapters written by healthcare professionals, researchers and patients. The perspectives flow from three primary themes: (a) the fundamental paradox between two primary drivers of our healthcare system, safety and cost, (b) the location of front-line workers in patient safety discussions, decisions and initiatives and (c) the need to approach patient safety from a systems perspective in acknowledgment that every detail of care, at every level, is interconnected.

The authors begin from the commonly held belief that patient safety is not progressing as quickly as expected, despite significant determination and effort. It seems that patient safety books and articles invariably contain a statement, something to the effect of, "It has been more than a decade since the Institute of Medicine's (2000) publication of To Err Is Human: Building a Safer Health System Called Us to Action," followed by examples of residual safety issues. The perspective suggests that a decade should surely have been enough time to fix most of what needed to be fixed. That point of view was likely put forward in this book as one of many paradoxes, ironies and challenges related to patient safety as another recurring theme. If one has the belief that patient safety should have progressed faster than it has, this book will probably change that perspective.

As with any discussion involving multiple perspectives, some outlooks will resonate more strongly than others. Depending on the situation of the reader with respect to his or her geography, role and experience with leading change to advance patient safety, he or she may dismiss some chapters as irrelevant. One of the primary themes explores the tensions between balancing cost and safety in a market model of healthcare in which multiple payers have differing priorities and coverage models aimed to control costs - not necessarily to improve safety. The market model paradox is examined from the model of healthcare in the United States, in which patients are situated as customers and healthcare providers become marketing agents whose aims are often directed towards customer satisfaction measures rather than actions grounded in the best interests of health and safety. It is claimed that this model interferes with professional ethics and, very likely, patient safety by forcing professional health services to be offered as competing product lines rather than individualized actions to meet patients' healthcare needs. Canadian readers, in a healthcare system funded primarily from one source, may be less familiar with these tensions. They will, however, be accustomed to making trade-offs arising from limited funding and quality agendas driven by systems-level, sometimes politically grounded priorities that compete 
directly with patient safety for limited resources and attention. The differently funded systems have unique stories that position patient safety against powerful, competing, externally driven priorities. Rather than dismissing seemingly foreign concepts, readers may instead reflect on parallel concepts that influence patient safety on more familiar ground.

The insufficiencies of information technology (IT) solutions to patient safety despite the highly anticipated value and enormous cost of IT and other electronic or mechanical solutions is a recurrent topic of discussion throughout the book. The poor integration of electronic data systems across the healthcare system, combined with mismatched user, administrator and vendor agendas, are highlighted as factors that limit the impact that could be achieved with better designed, better integrated and more user-determined systems. Hazards resulting from unintended effects, system limitations and user work-arounds are described.

There is a focus on the science of sleep deprivation as it relates to poor performance, poor concentration and employee health issues contrasted against the apparent disregard of that evidence with respect to physician and nursing work patterns by policy makers, administrators, educational systems and professional regulatory bodies. The roles that hierarchical, socio-economic, historical, preferential and other factors play in supporting persistently unhealthy and often unsafe hours and patterns of work are explored.

Seemingly, a patient safety book would not be complete without at least one reference to the aviation industry and its achievements in advancing its own safety agenda. This book doesn't disappoint, with intermittent references to Crew Resource Management (CRM), an aviation strategy that improves communication among crew members by levelling deeply engrained historical and hierarchical barriers to communication. What is different and more insightful in this book, compared to many, is the position that a CRM-like strategy for healthcare cannot be successfully transplanted from the aviation industry without first understanding how healthcare teams function from an insider (in the cockpit) perspective. The negative impact on patient safety resulting from poor communication embedded in systems and cultures that don't support any other kind of communication is extensively explored. The authors suggest that only a systematic study of team relationships, as done in the aviation industry to support CRM, will lead to the type of change required to systematically improve patient safety. The concept of team intelligence, extending from the idea of emotional intelligence (EI), adds to the dialogue of understanding and attending to the needs of teams to support better communication and a more integrated, inter-professional patient safety focus. 
It is acknowledged by several of the contributing authors that a paradigm shift from a top-down approach to one that is more inclusive of front-line staff, and in particular unions, is required to advance patient safety. Further, cultures that support patient safety are described as required both to reveal safety issues through voluntary reporting and to resolve them through insight that exists largely untapped in the hard-to-access private work spaces of front-line workers.

Leaders are challenged to think of patient safety from a broad systems perspective. The authors converge on the understanding that competing demands, limitations of available measures for indicators of importance, profound changes in all professions including but not limited to labour supply, economic restrictions and a market view on healthcare are likely to contribute to a default to quick-fix solutions. Short-sighted, narrowly conceived strategies based primarily on issues that are mandated and easy to measure are unlikely to achieve properly targeted patient safety priorities. The message is clear that patient safety is a continuous play of trade-offs against competing demands, including competing demands within the safety movement itself.

The book concludes with an insightful summary: 27 paradoxes, ironies and challenges to patient safety. The concluding chapter could stand on its own as a comprehensive list of complexities and peculiarities in our healthcare system that affect the progress of patient safety. It is enhanced by the unique perspectives in preceding chapters and collectively will contribute to better understandings of why patient safety shouldn't be a targeted destination within a preconceived timeframe. The book will advance an appreciation that patient safety involves nearly everything that we do in healthcare and that when we do it well, it is unremarkable because nothing happens. That idea is summarized in paradox number three, "Patient safety is not a thing, a single action, or an attitude; it's an outcome obscured by the paradox of prevention."

This book will be valuable to leaders in healthcare to reconcile tensions that exist with respect to the pace of improving patient safety within a complex system.

\section{References}

Institute of Medicine (IOM). 2000. To Err Is Human: Building a Safer Health System. Washington, DC: National Academies Press. 